\title{
Low Molecular Weight Heparin and Aspirin Prophylaxis after Total Knee Arthroplasty
}

\section{Sarzaeem MM ${ }^{\star}$}

Orthopedic surgeon, Imam Hossein Hospital, Tehran, Iran

*Corresponding author: Sarzaeem MM, Orthopedic surgeon, Imam Hossein Hospital, Tehran, Iran, Tel: +98 939029 22; E-mail: mmsarzaeem@yahoo.com

Rec date: October 15, 2014; Acc date: October 20, 2014; Pub date: October 22, 2014

Copyright: ( 2014 Sarzaeem MM, This is an open-access article distributed under the terms of the Creative Commons Attribution License, which permits unrestricted use, distribution, and reproduction in any medium, provided the original author and source are credited.

Keywords: LMWH; Aspirin prophylaxis; Total knee arthroplasty; Thromboprophylaxis

\section{Editorial}

Thromboembolic disease is a well-recognized complication after hip and knee arthroplasty. There is near universal agreement regarding the need for perioperative thromboprophylaxis. The exact incidence of deep venous thrombosis and pulmonary emboli is controversial. Incidence rates of more than $50 \%$ are reported without prophylaxis. With modern joint protocols using early mobilization, mechanical compression devices, and improved anesthetic techniques, the incidence has dramatically decreased. A recent review of 1179 cases by Dorr et al using a multimodal approach for thrombosis prevention reported a $0.25 \%$ rate of pulmonary emboli and $5.2 \%$ rate of deep venous thrombosis (only $0.4 \%$ that were clinically symptomatic). Even protocols using no chemical prophylaxis have shown a thrombosis rate of less than $5 \%$.

The optimal regimen would be safe, effective, and inexpensive. Such a regimen has been elusive. Modern hip and knee arthroplasty protocols emphasize early mobilization and mechanical compression devices. These methods alone have proven safe and beneficial. The major controversy remaining is the ideal chemoprophylaxis regimen. Conflicting recommendations from the American College of Chest Physicians (ACCP) and the American Academy of Orthopedic Surgeons (AAOS) have added to this dilemma. Pay for performance and medical liability must also be factored into the decision-making process of the practicing joint arthroplasty surgeon. Low-molecularweight heparin (LMWH) has demonstrated efficacy in preventing deep venous thrombosis in numerous clinical trials. LMWH that has rapid antithrombotic action, limited variability in its effects, and linear pharmacokinetics. It is advocated by both the AAOS and ACCP for chemical thromboprophylaxis after hip and knee arthroplasty. It is also approved by the Surgical Care Improvement Project and almost all oversight groups. The efficacy of LMWH is well documented. Concerns regarding safety do exist, and attempts by some centers to adhere to the ACCP guidelines have been met with increased bleeding and wound complications. The exact duration of treatment is also controversial. The increased cost especially with extended treatment regimens must be recognized. Orthopedic surgeons have some concern by using LMWH especially about wound complication. A lot of revision surgeries annually are done because of hematoma formation in surgical site, and sometimes may lead to joint infection which is a disaster. So it seems that using a chemoprophylaxis agent which is either effective or less harmful is a must. Aspirin (acetylsalicylic acid) has been advocated by multiple authors and is included in the AAOS guidelines and also recently approved by ACCP. Aspirin has shown benefit in preventing pulmonary emboli and is believed to be the safest and least expensive regimen. It was not recommended by the ACCP secondary to concerns regarding efficacy in preventing deep venous thrombosis in previous edition but in 2012 guideline, it has been approved. Historical data certainly raise concern regarding aspirin after hip and knee arthroplasty. More recent studies combining aspirin with modern surgical techniques and multimodal thromboProphylaxis protocols have shown very low rates of thromboembolic disease, when combined with mechanical compression devices and early mobilization, a low rate of symptomatic thromboembolic disease was noted. Therefore significant cost savings with a low complication rate can be achieved by changing from LMWH to Aspirin in joint replacements surgeries. 